Gut and Liver, Vol. 9, No. 3, May 2015, pp. 358-369

\title{
Nucleotide Binding Oligomerization Domain 1 Is an Essential Signal Transducer in Human Epithelial Cells Infected with Helicobacter pylori That Induces the Transepithelial Migration of Neutrophils
}

\author{
Beom Jin Kim ${ }^{*}{ }^{\dagger}$, Jae Yeol Kim*, Eung Soo Hwang ${ }^{\dagger}$, and Jae Gyu Kim* \\ ${ }^{*}$ Department of Internal Medicine, Chung-Ang University College of Medicine, Seoul, and ${ }^{\dagger}$ Department of Microbiology and Immunology, \\ Seoul National University College of Medicine, Seoul, Korea
}

Background/Aims: The cytosolic host protein nucleotide binding oligomerization domain 1 (Nod1) has emerged as a key pathogen recognition molecule for innate immune responses in epithelial cells. The purpose of the study was to elucidate the mechanism by which Helicobacter pylori infection leads to transepithelial neutrophil migration in a Nod1-mediated manner. Methods: Human epithelial cell lines AGS and Caco-2 were grown and infected with H. pylori. Interleukin (IL)-8 mRNA expression and IL-8 secretion were assessed, and nuclear factor $\kappa \mathrm{B}(\mathrm{NF}-\mathrm{\kappa B})$ activation was determined. Stable transfections of AGS and Caco-2 cells with dominant negative Nod1 were generated. Neutrophil migration across the monolayer was quantified. Results: Cytotoxin-associated gene pathogenicity island (cagPAl)(+) $\mathrm{H}$. pylori infection upregulated IL-8 mRNA expression and IL-8 secretion in AGS and Caco-2 cells compared with controls. NF- $\mathrm{KB}$ activation, IL-8 mRNA expression and IL-8 secretion by cagPAl knockdown strains were reduced compared with those infected with the wild-type strain. NF-אB activation, IL-8 mRNA expression and IL-8 secretion in dominant-negative (DN)-Nod1 stably transfected cells were reduced compared with the controls. The transepithelial migration of neutrophils in DN-Nod1 stably transfected cells was reduced compared with that in controls. Conclusions: Signaling through Nod1 plays an essential role in neutrophil migration induced by the upregulated NF-kB activation and IL-8 expression in H. pyloriinfected human epithelial cells. (Gut Liver 2015;9:358-369)

Key Words: Nod1; Helicobacter pylori; cag pathogenicity island; Neutrophils; Transepithelial migration

\section{INTRODUCTION}

Helicobacter pylori, a spiral, microaerophilic, Gram-negative bacteria that colonizes gastric epithelial cells. ${ }^{1-3} \mathrm{H}$. pylori is estimated to have infected more than half of all people worldwide. ${ }^{4,5} \mathrm{H}$. pylori is a major cause of chronic gastritis, gastric and duodenal ulcers, gastric carcinomas, and mucosa-associated lymphoid tissue lymphoma. ${ }^{3,6}$ The severity of $H$. pylori-related disease, however, varies greatly among infected individuals and seems to be influenced by both host and bacterial factors. ${ }^{5,7}$

To date, little is known about the nature of the H. pylori-induced proinflammatory signals and the intracellular signals directing the activation of immediate early response transcription factors. $H$. pylori strains containing a cluster of 31 genes known as the cytotoxin-associated gene pathogenicity island (cagPAI) are more frequently associated with severe gastric inflammation, ulceration and an increased risk of gastric cancer. ${ }^{8-10}$ Especially, the cagPAI genes are proposed to encode a type IV secretion apparatus which is thought to mediate the translocation of protein effector molecules into its target host cell. ${ }^{11}$ Given that a functional type IV secretion apparatus is required for H. pyloriinduced nuclear factor $\kappa \mathrm{B}(\mathrm{NF}-\kappa \mathrm{B})$ activation in gastric epithelial cells, it is suggested that an intracellular receptor may be involved in recognition of an $H$. pylori product that is presented in the cells.

Recently, the cytosolic host protein nucleotide binding oligomerization domain 1 (Nod1) has emerged as a key pattern recognition molecule (PRM) for innate immune responses in epithelial cells. ${ }^{12}$ This protein acts as an intracellular 'sensor' of bacterial pathogens through its recognition of cell wall peptidoglycan (PG). ${ }^{12}$ As a result of detailed molecular studies, human Nod1 was shown to exhibit exquisite specificity for a diami-

Correspondence to: Jae Gyu Kim

Department of Internal Medicine, Chung-Ang University College of Medicine, 84 Heukseok-ro, Dongjak-gu, Seoul 156-861, Korea

Tel: +82-2-6299-3147, Fax: +82-2-6299-1137, E-mail: jgkimd@cau.ac.kr

Received on June 14, 2013. Revised on February 14, 2014. Accepted on February 16, 2014. Published online on June 18, 2014

pISSN 1976-2283 eISSN 2005-1212 http://dx.doi.org/10.5009/gnl13218

(a) This is an Open Access article distributed under the terms of the Creative Commons Attribution Non-Commercial License (http://creativecommons.org/licenses/by-nc/3.0) which permits unrestricted non-commercial use, distribution, and reproduction in any medium, provided the original work is properly cited. 
nopimelate containing GlcNAc-MurNAc tripeptide motif that is almost exclusively found in Gram-negative bacterial PG. ${ }^{13}$ In addition, the dipeptide $\gamma$-D-glutamyl-meso-diaminopimelic acid was revealed as the key structures recognized by Nod $1 .{ }^{14}$ Later, Nod1 recognition of $H$. pylori PG was shown to result in $N F-\kappa B$ activation and subsequent interleukin (IL)-8 production in epithelial cells. ${ }^{5}$ However, the precise mechanism by which this extracellular pathogen is able to induce proinflammatory responses in gastric epithelial cells has remained obscure. Furthermore, little is known about the role of Nod1 as a signal transducer in $H$. pylori-infected gastric epithelial cells.

Neutrophil plays a central role in host defense by migrating to the site of infection and eliminating pathogen. The mechanisms underlying the movement of neutrophils from the bloodstreams to sites of infection are complex and involve numerous adhesion molecules, cytokines and chemoattractants that function to neutrophils. Especially, IL-8 is recognized as an important cytokine in transepithelial neutrophil migration of $H$. pylori infection. Therefore, the purpose of the present study is to elucidate the mechanism by which $H$. pylori leads to transepithelial neutrophil migration in a Nod1-mediated manner.

\section{MATERIALS AND METHODS}

\section{Cell lines and cell culture}

Human gastric epithelial cell line AGS (ATCC CRL 1739), human colon epithelial line Caco-2 (ATCC HTB-37) were cultured in DMEM and RPMI 1680 supplemented with 10\% fetal bovine serum (FBS) and $2 \mathrm{mM} \mathrm{L-glutamine} \mathrm{(Gibco,} \mathrm{Invitrogen,} \mathrm{Carls-}$ bad, CA, USA) in $5 \% \mathrm{CO}_{2}$ at $37^{\circ} \mathrm{C}$.

MKN-45, Kato III, and U-937 cells were cultured in DMEM and RPMI 1640 supplemented with 10\% FBS and $2 \mathrm{mM} \mathrm{L-glu-}$ tamine (Gibco, Invitrogen) in $5 \% \mathrm{CO}_{2}$ at $37^{\circ} \mathrm{C}$.

\section{Bacteria and infection protocols}

Clinically isolated cag(+) H. pylori strain, HP99, were provided from H.C. Jung (Seoul National University College of Medicine, Seoul, Korea). To examine the role of cagPAI, ATCC 60190 strain and its cagPAI knockdown H. pylori strains (CagA ${ }^{-}$and $\mathrm{CagE}^{-}$) were obtained from Y.C. Lee (Yonsei University College of Medicine, Seoul, Korea). Epithelial cells grown to confluent were infected with bacteria at a multiplicity of 200 .

\section{Generation of stably transfected cell lines with dominant- negative Nod1}

AGS and Caco-2 cells were stably transfected with dominantnegative (DN) Nod1 expression vector (pcDNA3-Nod1 $\triangle$ CARDmyc) or with control empty vector (pcDNA3) by using Lipofectamine Plus (Invitrogen, Carlsbad, CA, USA), respectively. pcDNA3-Nod1 $\triangle$ CARD-myc was provided by G. Nunez (University of Michigan, Ann Arbor, MI, USA).

Each of 0.2 and $0.5 \mathrm{mg} / \mathrm{mL}$ G418-resistant colonies were iso- lated by using glass cloning cylinders.

Production of DN-Nod1 in cells stably transfected with pcDNA3-Nod1 $\triangle$ CARD-myc was determined by immunoblotting with monoclonal anti-myc antibody.

\section{RNA extraction and reverse transcription-polymerase chain reaction}

Total cellular RNA was extracted with RNeasy mini kit (Qiagen, Valencia, CA, USA) and treated with RNase-free DNase to remove any contaminating genomic DNA and stored frozen at $-70^{\circ} \mathrm{C}$ until before using. Total RNA is reverse transcribed into cDNA using SuperScript ${ }^{\circledR}$ II Reverse Transcriptase (Invitrogen). For reverse transcription polymerase chain reaction (RT-PCR), 1 $\mu \mathrm{g}$ of total cellular RNA was reverse transcribed, and cDNA was amplified as described previously. ${ }^{15}$ The $\beta$-actin primers were sense primer $5^{\prime}$-TGACGGGGTCACCCACACACTGTGCCCATCTA-3' and antisense primer primer 5'-CTAGAAGCATTGCGGTGGACGATGGAGGG-3', and IL-8 primers were sense primer 5'-ATGACTTCCAAGCTGGCCGTGGCT-3' and antisense primer 5'-TCTCAGCCCTCTTCAAAAACTTCTC-3'. These sets of primers yielded PCR products that were 661 and 289 bp long, respectively. After a hot start, the amplification profile was $45 \mathrm{sec}-$ onds of denaturation at $95^{\circ} \mathrm{C}$ and 45 seconds of annealing and extension at $72^{\circ} \mathrm{C}$, and 1 minute of denaturation at $95^{\circ} \mathrm{C}$ and 2.5 minutes of annealing and extension at $60^{\circ} \mathrm{C}$ for 30 cycles, respectively. Negative control reaction mixtures contained no added RNA in the RT reaction mixtures and no cDNA in the PCR amplification mixtures.

\section{Real-time RT-PCR}

For real-time PCR, $1 \mu \mathrm{L}$ of cDNA was amplified by using an ABI Prism 7300 Real-Time PCR System (Applied Biosystems, Foster City, CA, USA) with $2 \times$ SYBR Green master mixture (Qiagen $\mathrm{GmbH}$, Hilden, Germany) as described previously. ${ }^{16,17}$ The $\beta$-actin primers were sense primer $5^{\prime}$-AAGATGACCCAGATCATGTT-3' and antisense primer primer 5'-GCGACATAGCACAGCTTCT- $3^{\prime}$, and IL-8 primers were sense primer 5'-ACATGACTTCCAAGCTGGCC-3' and antisense primer 5'-CAGAAATCAGGAAGGCTGCC-3'. A $25 \mu \mathrm{L}$ of mixture consisting $1 \mu \mathrm{L}$ of cDNA, $10 \mu \mathrm{L}$ of $2 \times$ SYBR Green master I mixture and primers was amplified 40 times repeatedly under 15 seconds in $95^{\circ} \mathrm{C}, 1$ minute in $60^{\circ} \mathrm{C}$ using 7300 Real-Time PCR System.

\section{Enzyme-linked immunosorbent assay}

To measure the secreted IL-8 on media, enzyme-linked immunosorbent assay (ELISA) was performed. ELISA plate was coated with monoclonal antihuman IL-8 antibody (Endogen, Boston, MA, USA) in a coating buffer (14.2 mM Na $\mathrm{CO}_{3}, 34.9$ $\mathrm{mM} \mathrm{NaHCO}_{3}, 3.1 \mathrm{mM} \mathrm{NaN}_{3}, \mathrm{pH}$ 9.6) and incubated overnight at room temperature. The plate was washed three times with phosphate-buffered saline (PBS) containing 0.05\% Triton X-100 and blocked with PBS containing 1\% bovine serum albumin (Sigma, 
St. Louis, M0, USA) in $0.05 \%$ Triton X-100 for 2 hours at $37^{\circ} \mathrm{C}$. Then the plate was washed again three times and biotin-labeled detecting antibody, and HRP-conjugated Streptavidin were added to each well incubated for 1 hour at $37^{\circ} \mathrm{C}$. After washing three times with PBS containing 0.05\% Triton X-100 color was developed by adding a TMB substrate solution to the wells. The absorbency at $405 \mathrm{~nm}$ was measured using an ELISA reader. ELISA was sensitive to Ab concentration of $8 \mathrm{ng} / \mathrm{mL}$.

\section{Western blotting}

The cells were twice washed with cold PBS and collected by scraping after PRO-PREPTM (Intron Biotechnology, Seongnam, Korea). Then the cells were lysed in ice for 30 minutes with lysis buffer. The lysates were clarified by centrifugation in 12,000 rpm, 30 minutes at $4^{\circ} \mathrm{C}$ and the supernatant was collected. The protein concentration was determined using the Bradford assay. Then 30 $\mu \mathrm{g}$ of protein from each sample were boiled for 5 minutes and separated by SDS-PAGE using 12\% separating gels. The protein was electrophoretically transferred to a Nitrocellulose Transfer Membrane (Whatman GmbH, Dassel, Germany) and blocked with 5\% BSA in TBS-T buffer (20 mM Tris base, $1.37 \mathrm{mM} \mathrm{NaCl}$, $0.05 \%$ Tween-20, $\mathrm{pH}$ 7.6) overnight at $4^{\circ} \mathrm{C}$. The membrane was incubated with anti-IкB- $\alpha$ antibody (Santa Cruz Biotechnolgy, Santa Cruz, CA, USA) and antiphospho-IкB- $\alpha$ antibody (Santa Cruz Biotechnology) for 2 hours. After washing with TBS-T three times, the membrane was incubated with HRP-conjugated secondary antibody in TBS-T at 1:2,000 dilution for 1 hour. The blots were processed with an enhanced chemiluminescence (ECL) kit (Santa Cruz Biotechnology) and were performed with BioImagin RAS 1000 plus (Fugifilm, Tokyo, Japan).

\section{Electrophoretic mobility shift assay}

Nuclear extracts and cytoplasmic extract were prepared with NEPER ${ }^{\circledR}$ Nuclear and Cytoplasmic Extraction Reagents (Pierce, Rockford, IL, USA). Probe labeling were performed with Biotin 3' End DNA Labeling Kit (Pierce) using NF- $\kappa \mathrm{B}$ consensus oligonucleotide, 5'-AGT TGA GGG GAC TTT CCC AGG C-3'. Nucleic extracts were obtained 60 minutes after infection. After extracted proteins were electrophoresis with $0.5 \mathrm{X}$ TBE in 6\% polyacrylamide gel, using LightShift Chemiluminescent Electrophoretic Mobility Shift Assay (EMSA) Kit (Pierce), it was transferred at nylon membrane. Then the membrane was cross-linked at transilluminator for 10 minutes and was developed with Bio-Imagin RAS 1000 plus.

\section{Monolayer epithelial culture and measurement of con- fluency}

Human colonic epithelial Caco-2 confluent monolayers were grown on Transwell ${ }^{\circledR}$ (Costar, Cambridge, MA, USA) with polycarbonate membrane ( $3.00 \mu \mathrm{m}$ pore size) coated cell matrix type I collagen (Nitta Gelatine, Tokyo, Japan) in Dulbecco's modified Eagle's medium. Cells were seeded onto inverted inserts and were allowed to attach overnight, after which the inserts were placed upright into 24-well culture plates, thus orienting the basolateral side of the monolayer upward. Confluency and integrity of monolayers was assessed by measuring the electrical resistance using Millicell-ERS (Millipore Corp., Bedford, MA, USA), which permits measurements on monolayers grown on inserts. We tested our system on cells cultured at everyday since these cells develop electrical resistances that increase with time of culture. We used monolayers that developed suitable transepithelial resistances. We obtained sufficient electrical resistance $\left(\geq 500 \mathrm{ohm} \mathrm{m}^{2}\right)$ when Caco2 cells were cultured for more than 2 weeks. Subsequently, epithelial transmigration of neurtophil was induced using confluency and integrity of monolayers epithelial cell culture.

\section{Preparation of neutrophils}

Citrated venous blood from healthy human was separated into a granulocyte-rich fraction by Ficoll (Hitopaque; Sigma, St. Louis, MO, USA) and dextran-sedimentation techniques. Residual erythrocytes were eliminated by hypotonic lysis in $0.2 \%$ sodium chloride. This resulted in a cell fraction containing approximately more than 90\% neutrophils with 98\% to 99\% viability as determined from trypan blue dye exclusion.

\section{Measurement of neutrophil migration}

HP99 strains were infected for more than 24 hours in Caco2 cells. $1 \times 10^{6}$ cell $/ \mathrm{mL}$ of neutrophils were added to Caco- 2 cells in the presence of HP99, where bacteria were still infected in Caco-2 cells. We assessed the interaction between neutrophil and Caco-2 cells by checking electrical resistance at $0,1,2,4$, 8, and 24 hours after infection. The number of transmigrating neutrophil was quantified by Neubauer hemocytometers (VWR International, Lutterworth, UK). The cover glass was applied carefully so that diffraction patterns could be seen where the cover glass sat on the chamber body. The neutrophils trapped in the suspension was then applied and allowed to fill the chamber. After allowing the neutrophils to settle into one focal plane, we counted the number in the whole grid.

\section{Statistical analysis}

The Student t-test was used for numerical data, whereas the Mann-Whitney U-test was used for categorical data as appropriate. Data were analyzed using one-way analysis of variance for multiple comparisons. $p$ values of $<0.05$ were considered statistically significant.

We obtained the Institutional Review Board permission for blood sampling from Chung-Ang University Hospital (C2014026).

\section{RESULTS}

\section{1. cag(+) H. pylori infection increases IL-8 mRNA levels and IL-8 protein release in human gastric (AGS) and colon epithelial cell lines (Caco-2)}

First, we determined the expression of Nod1 and Nod2 in 
three human gastric epithelial cell lines (AGS, MKN-45, and Kato III) and U-937. As a result, Nod1 proved to be expressed in gastric epithelial cells and U-937. However, Nod2 was hardly expressed in the AGS cells (Fig. 1). Therefore, AGS cells are suitable for verifying the role of Nod1. Subsequently, human gastric epithelial cells AGS and human colonic epithelial cells Caco-2 were infected with cag(+) H. pylori strain, HP99. We performed qualitative RT-PCR to examine the IL-8 mRNA expression in HP99-infected AGS cells.

As shown in Fig. 2, incubation of AGS cells with HP99 re-

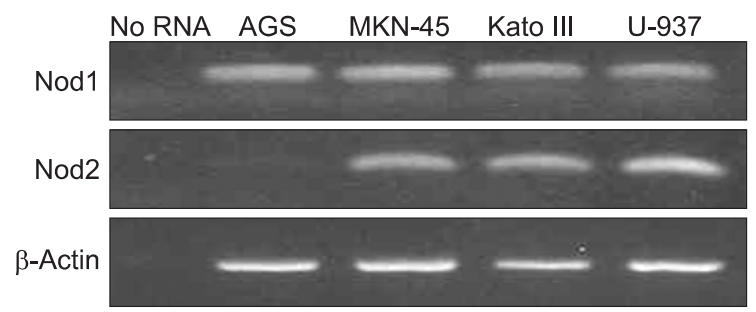

Fig. 1. Expression of nucleotide binding oligomerization domain 1 (Nod1) and Nod2 in AGS, MKN-45, Kato III, and U-937 cell lines. Nod1 was expressed in three gastric epithelial cells and U-937 cells. However, Nod2 was expressed at very low levels in AGS cells. sulted in the increased expression of IL-8 mRNA compared with those in controls by 4 hours after infection. IL-8 mRNA expression and secretion of IL-8 protein into the culture medium were confirmed by the real-time RT-PCR and by ELISA. As assayed by real-time PCR, IL-8 mRNA expression in AGS cells in response to infection with HP99 was significantly increased within the first 4 hours after infection (Fig. 2B). In addition, infected cells responded to produce a mean level in excess of $500 \mathrm{pg} / \mathrm{mL}$ of IL-8 by ELISA (Fig. 2C).

As shown in Fig. 3, incubation of Caco-2 cells with HP99 also resulted in the increased expression of IL-8 mRNA by PCR (Fig. 3A) and real-time RT-PCR (Fig. 3B), respectively. During the coincubation, IL-8 secretion by Caco- 2 cells infected with HP99 reached high value at approximately 4 hours of coincubation, and remained high during further coincubation. Especially, IL-8 secretion by Caco-2 cells infected with HP99 was rapid but weak when compared with AGS cells by ELISA (Fig. 3C).

\section{Components of the cagPAI is fundamental for IL-8 induc- tion in epithelial cells}

The role of cagPAI was examined comparing wild type $H$. pylori strain (ATCC 60190) with cagPAI knockdown H. pylori

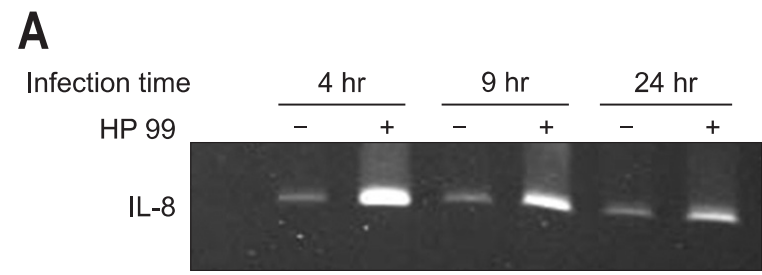

\section{B}

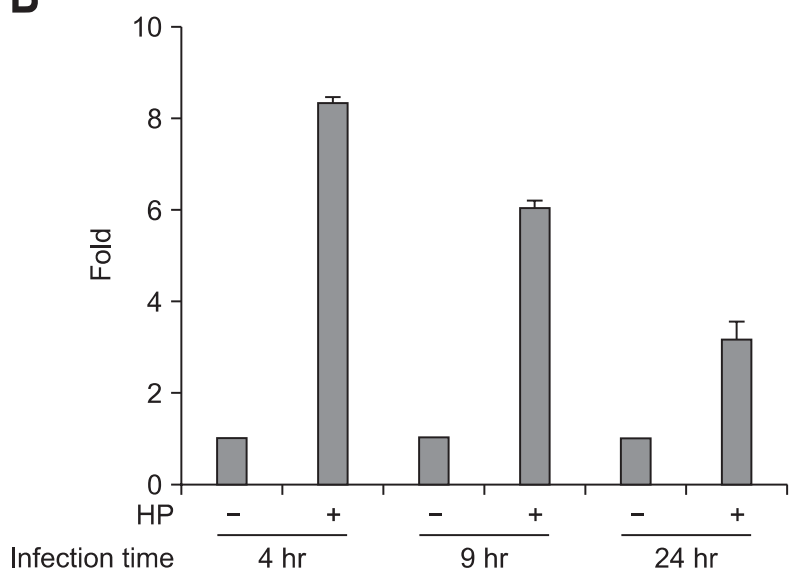

C

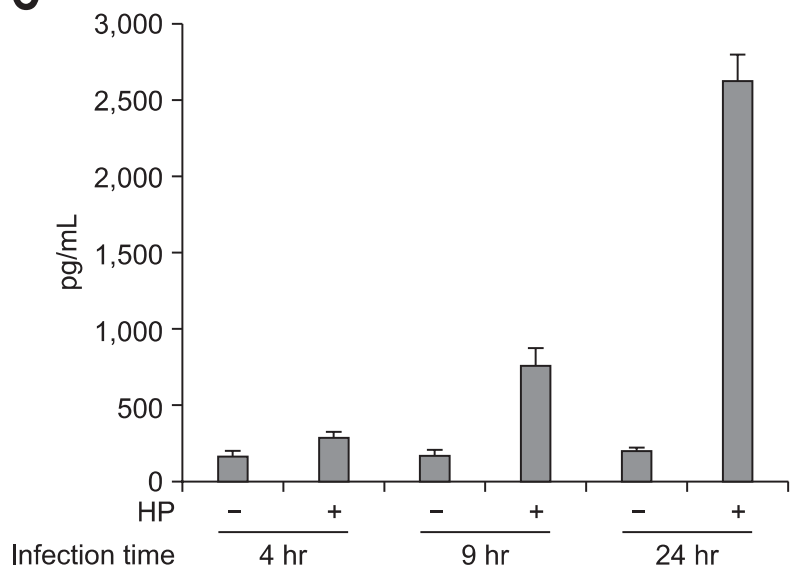

Fig. 2. Interleukin (IL)-8 mRNA expression and IL-8 production in AGS cells infected with cytotoxin-associated gene pathogenicity island (cagPAI) (+) Helicobacter pylori (HP99). (A) As assayed by qualitative reverse transcription-polymerase chain reaction (RT-PCR), the incubation of AGS cells with HP99 resulted in increased IL-8 mRNA expression compared with that in controls by 24 hours after infection. (B) IL-8 mRNA expression in response to infection with HP99 was significantly increased at 4 hours after infection, as determined by real-time RT-PCR. (C) As determined by enzyme-linked immunosorbent assay, infected cells produced IL-8 at a mean level in excess of $500 \mathrm{pg} / \mathrm{mL}$. The error bars indicate the standard error of the mean of triplicate samples, which were representative of three independent experiments. 


\section{A}

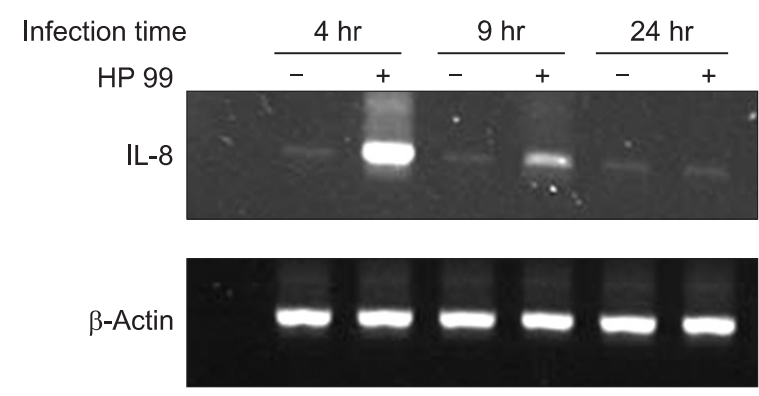

B

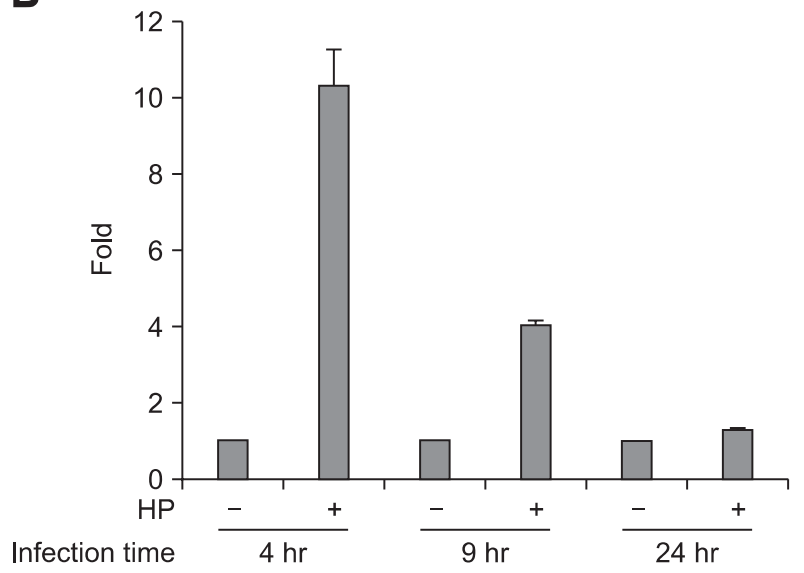

C

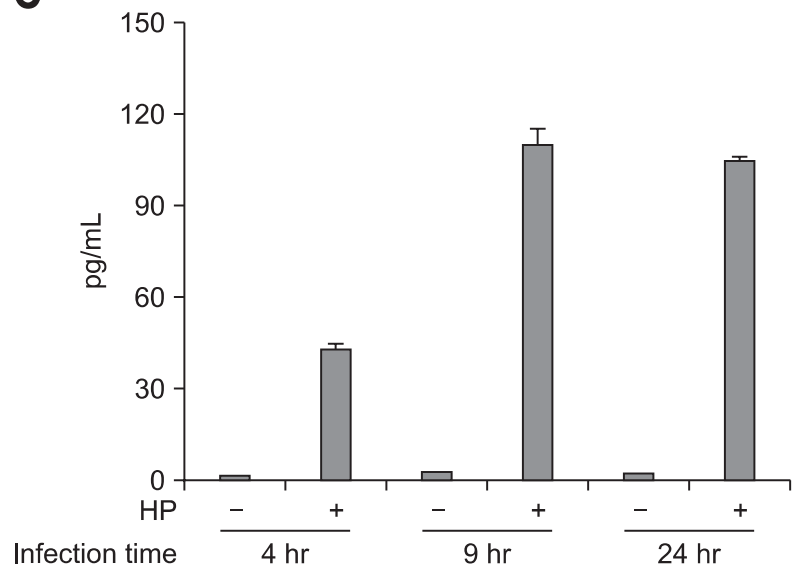

Fig. 3. Interleukin (IL)-8 mRNA expression and IL-8 production in Caco-2 cells infected with HP99. (A) As assayed by qualitative reverse transcription-polymerase chain reaction (RT-PCR), the incubation of Caco-2 cells with HP99 resulted in the increased expression of IL-8 mRNA compared with that in controls 24 hours after infection. (B) The incubation of Caco-2 cells with HP99 resulted in the increased expression of IL-8 mRNA by real-time RT-PCR. (C) During the coincubation, IL-8 secretion by Caco-2 cells infected with HP99 reached high values at approximately 4 hours of coincubation and remained high during further coincubation, up to 24 hours. The error bars indicate the standard error of the mean of triplicate samples, which were representative of three independent experiments.

strain (CagA ${ }^{-}$and $\mathrm{CagE}^{-}$). To assay IL-8 mRNA expression in wild type $H$. pylori-infected compared with cagPAI knockdown H. pylori, IL-8 mRNA levels were determined by real-time PCR. As a result, IL-8 mRNA levels were decreased by cagPAI knockdown strains than wild type $H$. pylori in AGS cells at 5 hours after infection (Fig. 4A). As shown in Fig. 4B, IL-8 mRNA levels were higher in the wild type $H$. pylori-infected cells than cagPAI knockdown $H$. pylori-infected cells by 12 - to 14 -fold. We tested the wild type and cagPAI knockdown $H$. pylori for their ability to induce IL-8 release from AGS cells. As a result of ELISA, knockdown mutants displayed a reduced ability to mediate IL-8 release, compared with the wild type (Fig. $4 \mathrm{C}$ ). The NF- $\kappa \mathrm{B}$ activation by cagPAI knockdown $H$. pylori infection was monitored

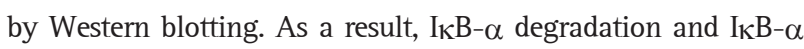
phosphorylation were reduced in the AGS cells infected with cagPAI knockdown $H$. pylori compared with those infected with wild type (Fig. 4D). Comparison of NF- $\kappa \mathrm{B}$ activation between cagPAI knockdown strains and wild type strain in AGS cells was made by EMSA. As a result, NF- $\kappa$ B binding in AGS cells infected with cagPAI knockdown strains were reduced compared with that in those infected with wild type strain (Fig. 4E).

\section{Nod1-mediated intracellular signaling induces NF-KB ac- tivation and promotes IL-8 production}

To investigate whether Nod1 might be involved in cagPAI-dependent recognition of $H$. pylori, we established Nod1-deficient cell lines by stable transfection of DN-Nod1 construct into AGS cells. To assay IL-8 mRNA expression in DN-Nod1 stably transfected AGS cells, IL-8 mRNA levels were determined by qualitative PCR. As a result, IL-8 mRNA expression in these cells were reduced compared with those in controls (Fig. 5A). As shown in Fig. 5B, decreased expression of IL-8 mRNA in DN-Nod1 stably trasfected AGS cells was observed in real-time RT-PCR assay. In order to determine whether Nod1 regulates IL-8 secretion, DN-Nod1 stably transfected AGS cells along with the controls were stimulated with HP99, and cell culture supernatants were collected. At 4 hours after infection, we observed approximately 4-fold induction of IL-8 in DN-Nod1 stably transfected AGS cells, compared with approximately 18-fold in the control vector transfected cells (Fig. 5C). Western blotting showed that $\mathrm{I}_{\kappa} \mathrm{B}-\alpha$ degradation and $\mathrm{I}_{\kappa} \mathrm{B}-\alpha$ phosphorylation were reduced in DN-Nod1 stably transfected AGS cells compared with those in 


\section{A}
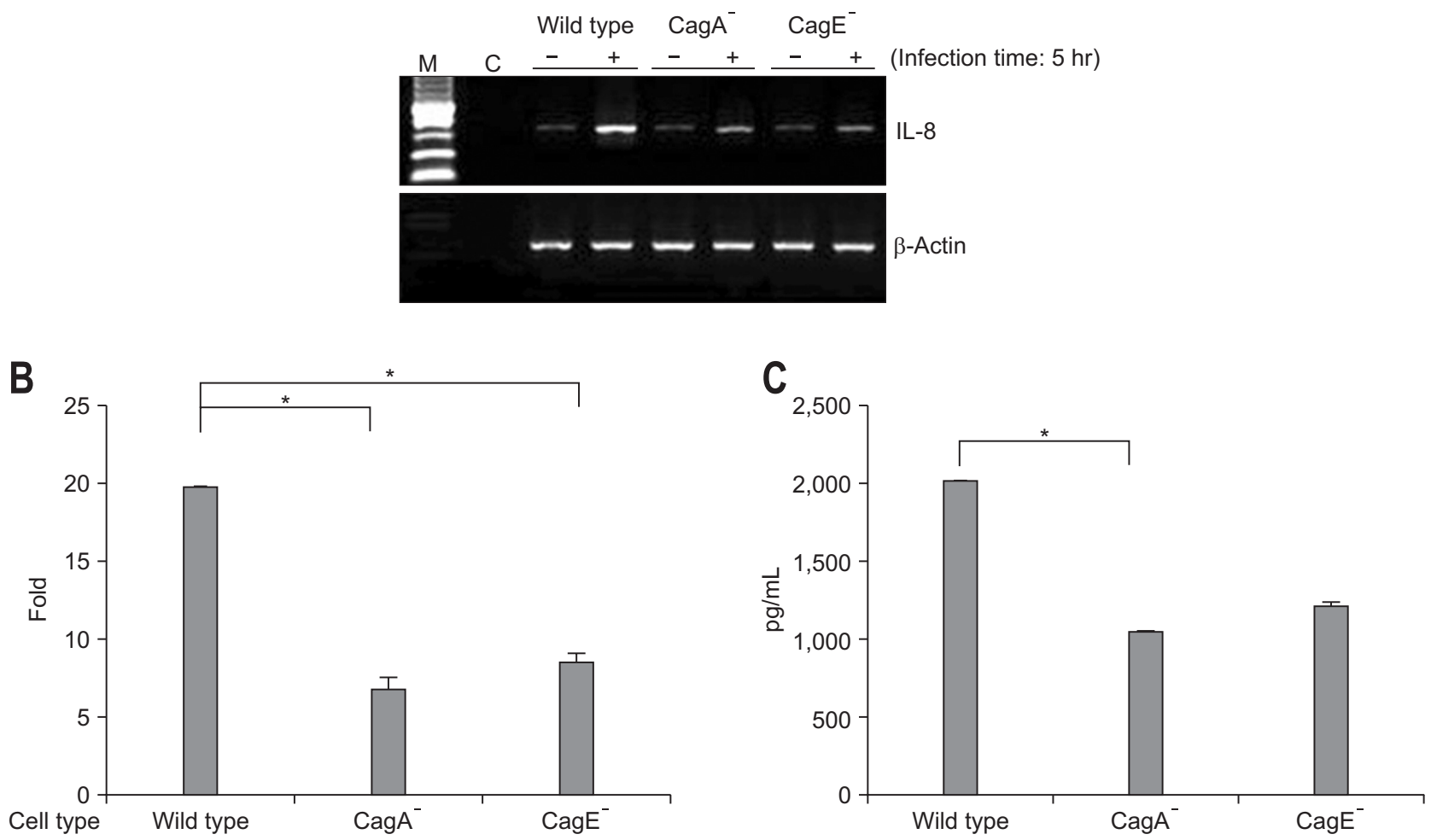

D

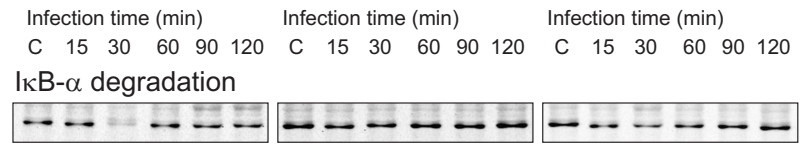

IКB- $\alpha$ phosphorylation
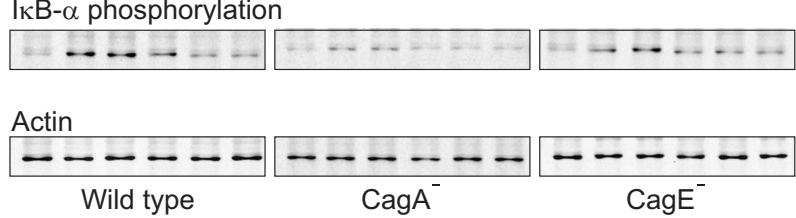

E

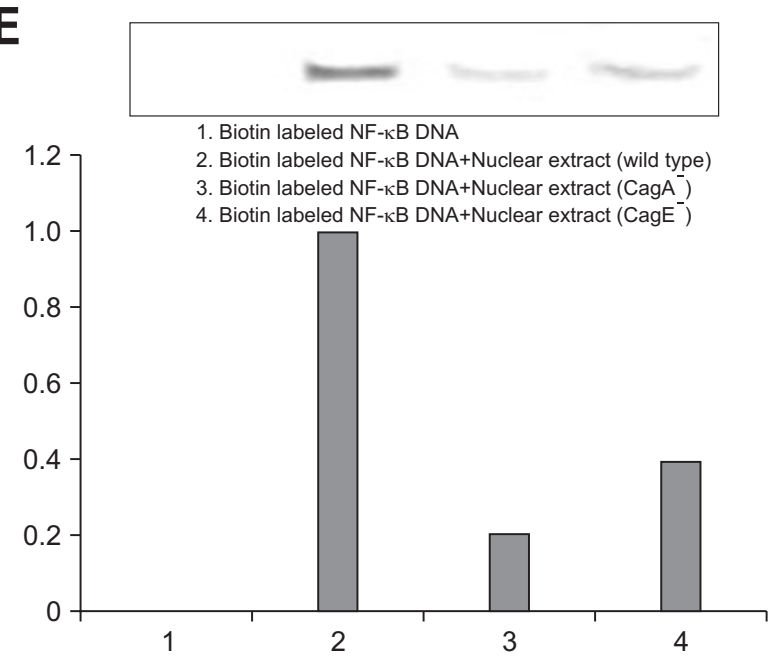

Fig. 4. Interleukin (IL)-8 mRNA and protein expression in AGS cells infected with wild-type (ATCC 60190) and cytotoxin-associated gene pathogenicity island (cagPAI)-depleted (CagA ${ }^{-}$and $\mathrm{CagE}^{-}$) Helicobacter pylori. (A) IL-8 mRNA expression was decreased by infection with cagPAI knockdown strains compared to wild-type $H$. pylori in AGS cells at 5 hours after infection. (B) The IL-8 mRNA levels were significantly higher in the wild-type $H$. pylori-infected cells than in the cagPAI-depleted $H$. pylori-infected cells. (C) The knockdown mutants displayed a reduced capacity to mediate IL-8 release compared with the wild-type strain. (D) I $\kappa \mathrm{B}-\alpha$ degradation and I $\kappa \mathrm{B}-\alpha$ phosphorylation were reduced in the AGS cells infected with cagPAI-depleted $H$. pylori compared with those infected with the wild-type strain. (E) Nuclear factor $\kappa B$ (NF- $\kappa B$ ) binding in AGS cells infected with the cagPAI knockdown strain was reduced compared with binding in cells infected with the wild-type strain. The error bars indicate the standard error of the mean of triplicate samples, which were representative of three independent experiments. ${ }^{*} \mathrm{p}<0.05$.

controls (Fig. 5D). To evaluate Nod1-dependent NF- $\mathrm{B}$ activation, we performed EMSA. As shown in Fig. 5E, NF- $\kappa \mathrm{B}$ binding in DN-Nod1 stably transfected AGS cells infected with HP99 was reduced compared with those in controls.

As shown in Fig. 6, DN-Nod1 stably transfected Caco-2 cells were infected with HP99, followed by PCR, real-time RT-PCR, and ELISA. Similar to the response in DN-Nod1 stably transfected AGS cells, IL-8 mRNA expression and IL-8 secretion in DN Nod1-transfected Caco-2 cells were reduced compared with those in control, respectively (Fig. 6). 


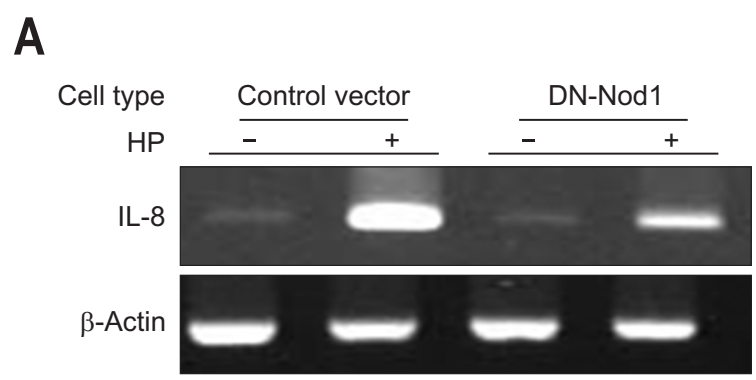

B

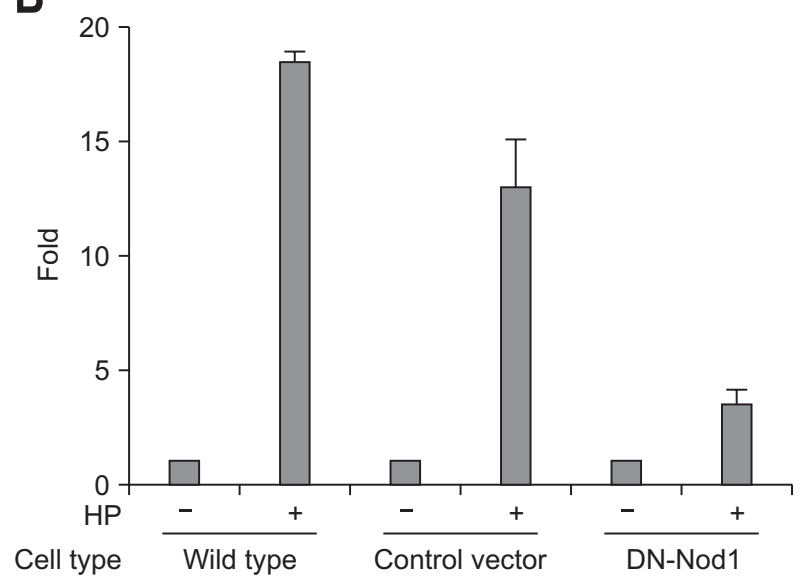

D

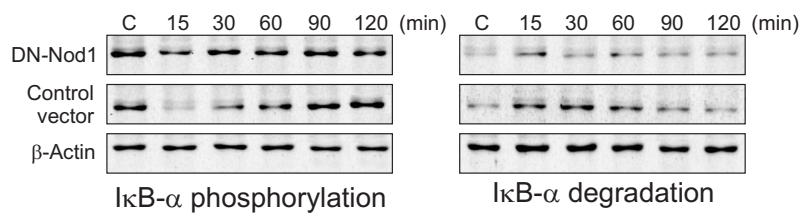

C

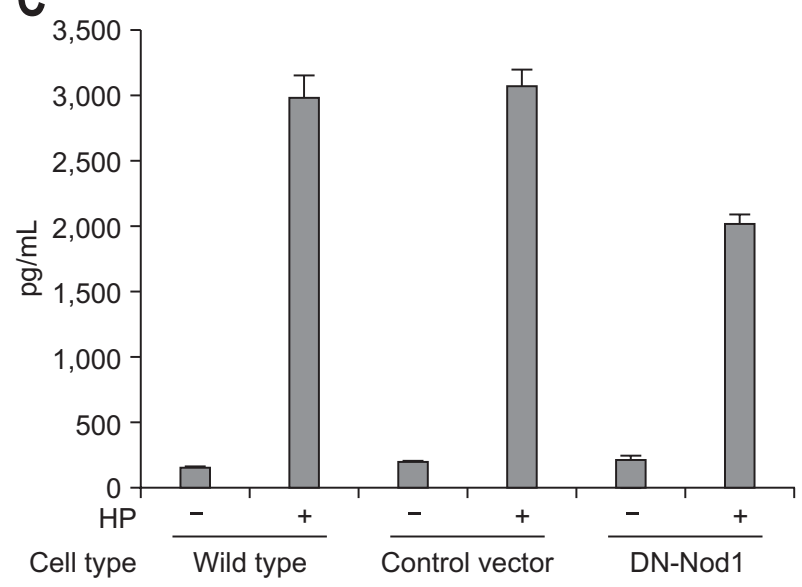

E

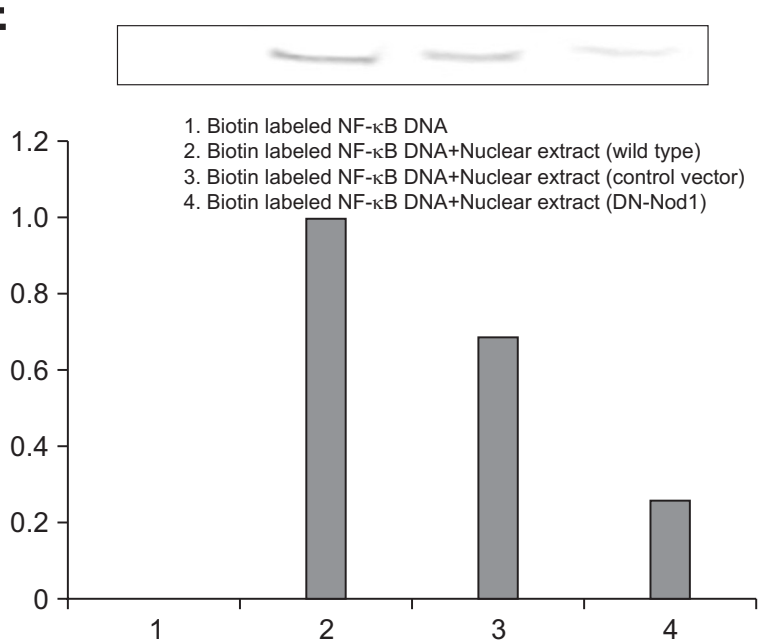

Fig. 5. Interleukin (IL)-8 mRNA expression and IL-8 production in dominant-negative nucleotide binding oligomerization domain 1 (DN-Nod1) stably transfected AGS cells infected with HP99. (A) IL-8 mRNA expression in DN-Nod1 stably transfected AGS cells was reduced compared with that in the controls. (B) At 4 hours after infection, IL-8 mRNA expression in DN-Nod1 stably transfected AGS cells was reduced compared with the control vector-transfected cells, as determined by real-time reverse transcription-polymerase chain reaction (RT-PCR). (C) IL-8 secretion by the DN-Nod1 stably transfected AGS cells was reduced compared with the control vector-transfected cells. (D) I $\mathrm{B}-\alpha$ degradation and I $\kappa \mathrm{B}-\alpha$ phosphorylation were reduced in the DN-Nod1 stably transfected Caco-2 cells compared with the controls. (E) Nuclear factor $\kappa \mathrm{B}$ (NF- $\kappa \mathrm{B}$ ) binding in the DN-Nod1 stably transfected Caco-2 cells was reduced compared with that in the controls. The error bars indicate the standard error of the mean of triplicate samples, which were representative of three independent experiments.

Control vector, pcDNA3.

\section{Nod1 determines the activation of IL-8 production inde- pendently of the cagPAI}

As shown in Fig. 7B, decreased expression of IL-8 mRNA in DN-Nod1 stably trasfected AGS cells infected with cagPAI knockdown strain was observed in real-time RT-PCR assay. In addition, ELISA showed that DN-Nod1 stably trasfected AGS cells infected with cagPAI knockdown strain displayed a reduced ability to mediate IL-8 release, compared with those infected with wild type ATCC 60190 (Fig. 7C). 

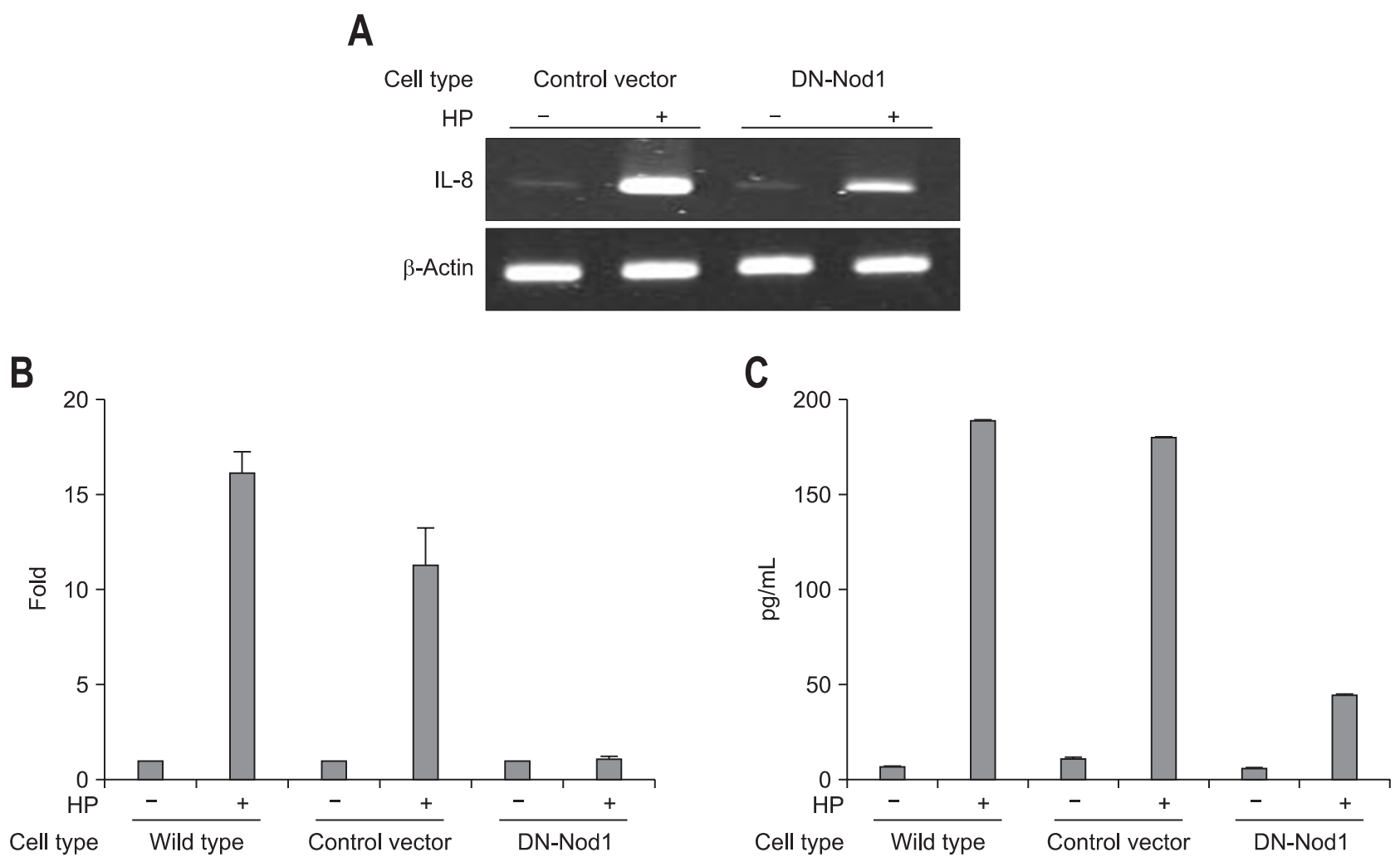

Fig. 6. Interleukin (IL)-8 mRNA expression and IL-8 production in dominant-negative nucleotide binding oligomerization domain 1 (DN-Nod1) stably transfected Caco-2 cells infected with HP99. (A) IL-8 mRNA expression in DN-Nod1 stably transfected Caco-2 cells was reduced compared with that in the controls. (B) IL-8 mRNA expression in DN-Nod1 stably transfected Caco-2 cells was reduced compared with that in the controls, as determined by real-time reverse transcription-polymerase chain reaction (RT-PCR). (C) IL-8 secretion by the DN-Nod1 stably transfected Caco2 cells was reduced compared with that in controls. The error bars indicate the standard error of the mean (SEM) of triplicate samples, which were representative of three independent experiments.

Control vector, pcDNA3.

\section{Nod1-mediated intracellular signaling induces transepi- thelial migration of the neutrophils}

In this study, proper resistance was defined as in case of $\geq 500 \Omega \mathrm{m}^{2}$ in Caco-2 monolayer. The change of the electrical resistance in DN-Nod1 stably transfected Caco-2 cells was lower than that in control vector transfected Caco-2 cells (Fig. 8). Quantification of transepithelial migration of neutrophil after HP99 infection in polarized inverted Caco-2 monolayer showed that neutrophil migration in DN-Nod1 transfected Caco-2 cells was reduced compared with that in control vector transfected Caco-2 cells (Fig. 9).

\section{DISCUSSION}

These experiments demonstrate that cagPAI-dependent $H$. pylori recognition can be mediated by intracytoplasmic signaling via NOD1. This resulted in induction of neutrophil migration through NF- $\mathrm{B}$ activation and subsequent IL-8 production.

Induction of the host epithelial cell proinflammatory gene program is essential for rapid activation of the host mucosal inflammatory response and is important for bacterial clearance and host survival following infection with enteric pathogens. ${ }^{18}$ However, no direct evidence for the link between induction of proinflammatory gene expression by $H$. pylori and a host antimicrobial response has been established so far. To characterize the regulated expression of IL-8 in response to H. pylori infection, we used the human gastric epithelial cell line, AGS as well as human colonic epithelial cell line, Caco-2. Gastric cell lines derived from adenocarcinomas are extensively used as model systems for the study of microbial pathogenesis. ${ }^{2}$ Especially, AGS cell line is known to provide useful models of the interaction between $H$. pylori and the gastric mucosa, and have been shown to respond to cagPAI(+) $H$. pylori in a Nod1-dependent manner. ${ }^{19,20}$ Consistent with previous reports, this study presented that AGS cells were compatible with Nod1 expression.

It has been known that $H$. pylori strains harboring a T4SS, encoded by the cagPAI, induce greater levels of NF- $\mathrm{KB}$ activation and IL-8 secretion than cagPAI lacking strains. ${ }^{7,21,22}$ Nevertheless, the exact mechanism by which PG may enter the host cell via the actions of the T4SS, so as to initiate these Nod1-dependent responses, has remained elusive. Therefore, the present study intended to demonstrate that the Nod1 stimulates to an increased activation of $\mathrm{NF}-\kappa \mathrm{B}$ downstream and a greater degree 


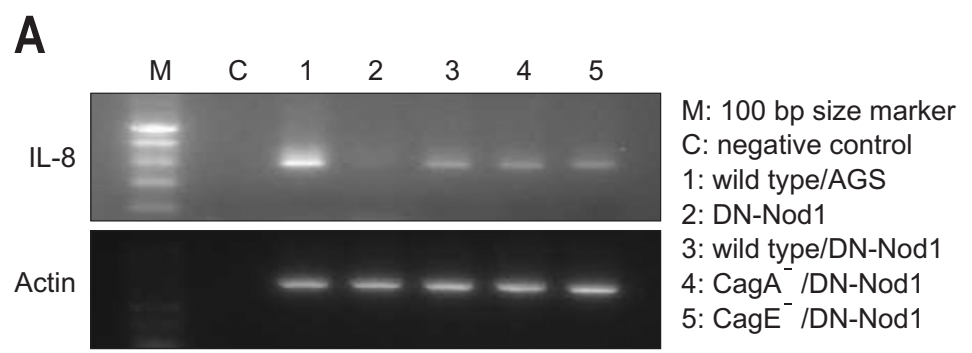

B

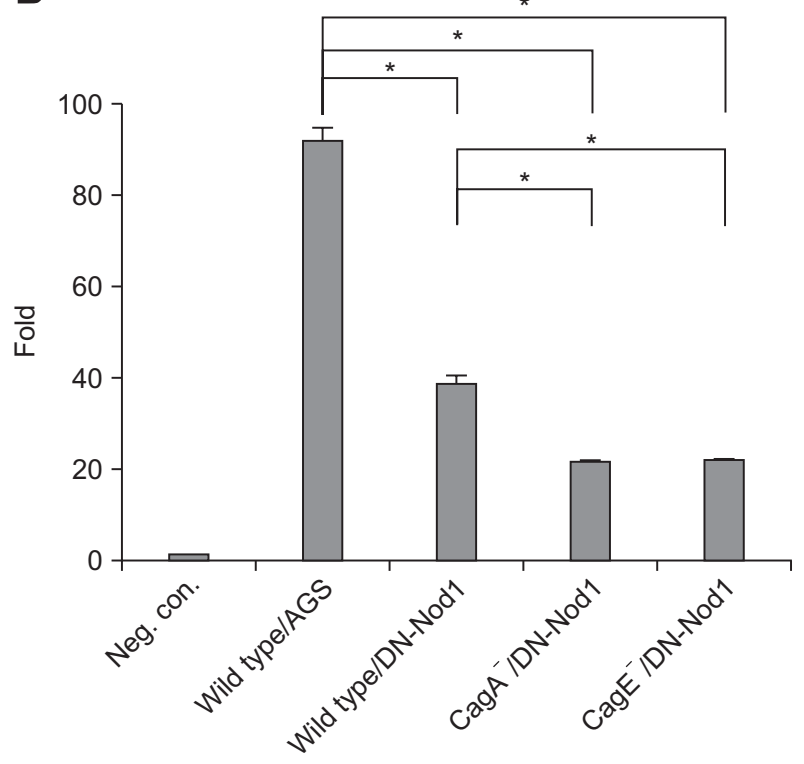

C

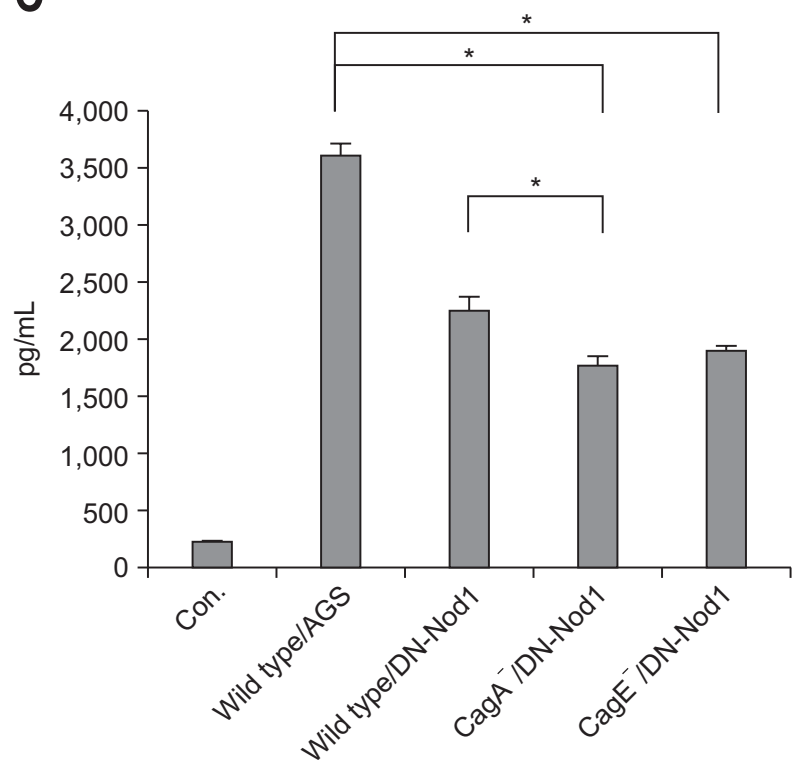

Fig. 7. Interleukin (IL)-8 mRNA and protein expression in dominant-negative nucleotide binding oligomerization domain 1 (DN-Nod1) stably transfected AGS cells infected with cytotoxin-associated gene pathogenicity island (cagPAI)-depleted Helicobacter pylori. (A) Decreased expression of IL-8 mRNA in DN-Nod1 stably transfected AGS cells infected with the cagPAI knockdown strain was observed compared with the expression levels in cells infected with wild-type ATCC 60190. (B) IL-8 mRNA expression in DN-Nod1 stably transfected AGS cells infected with cagPAIdepleted $H$. pylori was reduced compared with the expression in cells infected with wild-type ATCC 60190, as determined by real-time reverse transcription-polymerase chain reaction analysis. (C) DN-Nod1 stably transfected AGS cells infected with the cagPAI knockdown strain displayed a reduced capacity to mediate IL-8 secretion compared with cells infected with wild-type ATCC 60190. The error bars indicate the standard error of the mean of triplicate samples, which were representative of three independent experiments.

${ }^{*} \mathrm{p}<0.05$.

of mediation of inflammation. As a result, this study revealed that cagPAI(+) H. pylori activated gastric epithelial cell $\mathrm{NF}-\kappa \mathrm{B}$, leading to upregulation of IL-8 mRNA transcription and IL-8 protein production. It was also evident in colonic epithelial cells Caco-2.

Infection with a cagPAI(+) H. pylori strain causes a higher grade of gastric mucosal inflammation than an infection caused by a negative strain. ${ }^{3,7,23,24}$ This led us to determine whether cag$\mathrm{PAI}(+)$ and cagPAI knockdown strains of $H$. pylori differ in their ability to activate epithelial cell inflammatory responses. As a

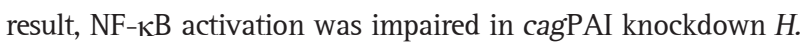
pylori-infected AGS cells compared with cagPAI(+) H. pyloriinfected AGS cells. Considering NF- $\kappa$ B activation actually lead to increased proinflammatory cytokine release, we analyzed the IL-8 release by AGS cells in response to $H$. pylori wild type and the cagPAI knockdown strains, respectively. As expected, cagPAI knockdown $H$. pylori did not induce IL-8 production so much as wild type $H$. pylori induced IL-8. Thus, the reduced IL-8 release from cagPAI knockdown $H$. pylori-infected AGS cells corroborates the observation that cagPAI mutant strains induce weak activation of transcription factors. ${ }^{10}$

Nod1 is thought to function in innate and adaptive immunity that determines the balance between health and disease. Recognition of $H$. pylori PG activates signaling cascades that culminate in the secretion of proinflammatory mediators, including chemokines and antimicrobial peptides. The process of $H$. pylori transferring its PG into the host epithelial cell, and its subsequent recognition by Nod1, results in the activation of NF- $\kappa B$ and the production of IL-8. ${ }^{3,6,21}$ In order to investigate the NF$\kappa \mathrm{B}$ activation by Nod1 in AGS and Caco-2 cells, we generated stably transfected DN-Nod1 cell lines. To further characterize cell responses in DN-Nod1 stably transfected AGS and Caco-2 cells, we ascertained that DN-Nod1 stably transfected AGS and Caco-2 cells were poorly responsive to cagPAI(+) $H$. pylori and 


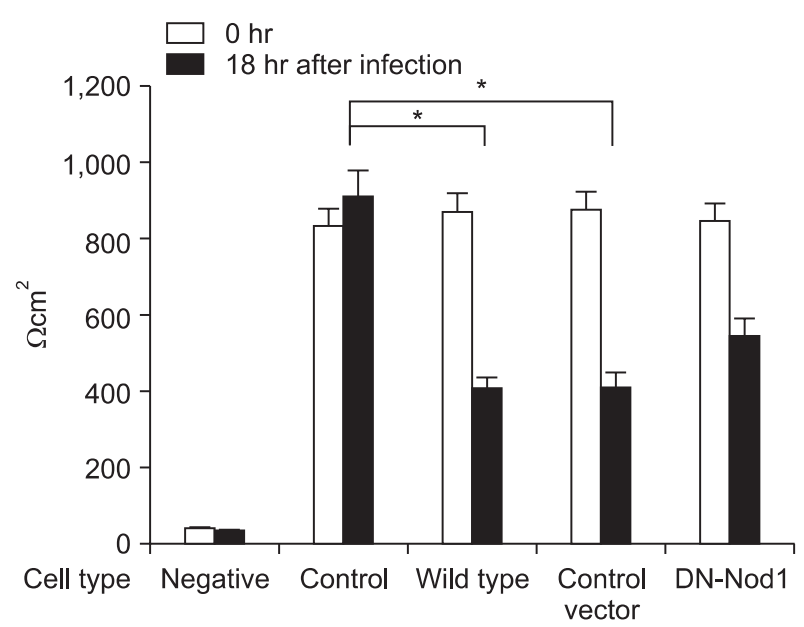

Fig. 8. Transepithelial electrical resistance in wild-type control vector-transfected cells and dominant-negative nucleotide binding oligomerization domain 1 (DN-Nod1) stably transfected Caco-2 cells. The change in the electrical resistance of DN-Nod1 stably transfected Caco-2 cells was lower than that of the control vector-transfected Caco-2 cells. The error bars indicate the standard error of the mean (SEM) of quadruplicate samples, which were representative of four independent experiments.

Control vector, pcDNA3. ${ }^{*} \mathrm{p}<0.05$.

resulted in reduced IL-8 production. Especially, NF- $\kappa \mathrm{B}$ binding was severely impaired in DN-Nod1 stably transfected AGS and Caco-2 cells with cagPAI(+) H. pylori. These data indicate that the Nod1 signaling pathway is significantly impaired in DNNod1 stably transfected AGS and Caco-2 cells. We tested the immune responses under cagPAI-/Nod1-independent condition in H. pylori infection. As shown in Fig. 7, DN-Nod1 stably transfected AGS cells infected with cagPAI knockdown strain did not show significant reduction in IL-8 production, compared with those infected with wild type strain. This indicates that cagPAI mutant strains also induce weak activation of $N F-\kappa B$ and subsequent IL-8 production under condition where Nod1 does not work. Furthermore, this phenomenon suggests the existence of another effector molecules which act effectively on the intracellular signal transduction. Actually, PG present in $H$. pylori-derived outer membrane vesicles was described to

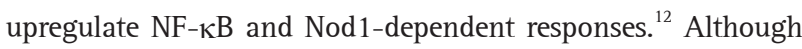
interactions between host cells and bacterial vesicles have been described in vitro, details are still poorly understood. ${ }^{4}$

Based on the results, we assume that NF- $\kappa \mathrm{B}$ activation in $H$. pylori-infected epithelial cells depends on Nod1, which occupies a key position in a signal transduction pathway. The neutrophil transmigration assay using inverted cell culture monolayers of polarized cells has been described. In many previous studies human colon epithelial cell lines, especially Caco-2 monolayer are suitable for intestinal epithelial transport model system..$^{15,25,26}$ Unfortunately, there is no suitable gastric epithelial monolayer yet. Actually, our attempts to confirm the formation and its confluence of monolayer using gastric epithelial cell line AGS

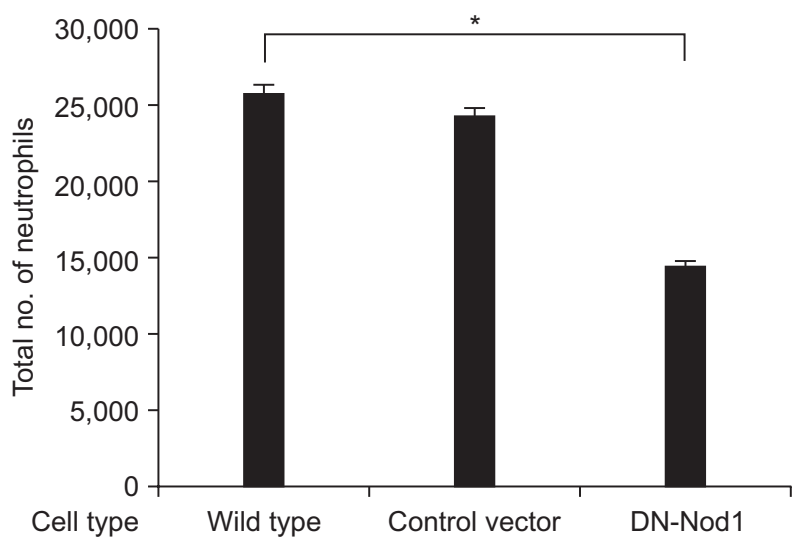

Fig. 9. Transepithelial neutrophil migration during HP99 infection in wild-type control vector-transfected cells and dominant-negative nucleotide binding oligomerization domain 1 (DN-Nod1) stably transfected Caco-2 cells. The quantification of transepithelial migration of neutrophils after HP99 infection in a polarized inverted Caco-2 monolayer showed that neutrophil migration in DN-Nod1 transfected Caco-2 cells was reduced compared with that in control vectortransfected Caco-2 cells. The error bars indicate the standard error of the mean (SEM) of quadruplicate samples, which were representative of four independent experiments.

Control vector, pcDNA3. ${ }^{*} \mathrm{p}<0.05$.

cells have not been successful (data not shown).

Invasion by microbial pathogens at mucosal surfaces elicits the infiltration of neutrophils, which subsequently use potent effector mechanisms including inflammatory mediators to eliminate pathogens. A consequence of the innate inflammatory response at mucosal surfaces influneces in the infiltration of neutrophils from the bloodstream to the epithelium. Neutrophils travel across this epithelial barrier into the lumen in order to confront colonizing pathogenic bacteria. The present study demonstrated that signaling pathway through Nod1 might act as a regulator of neutrophil migration in resistance and permeability of epithelial layer. It also indicates that transepithelial neutrophil migration is under the control of the Nod1-mediated intracellular signaling pathway. The reduced neutrophil migration in DN-Nod1 stably transfected cells indicates that the transcriptional activation of IL-8 and IL-8 secretion by the cells might be directly correlated with activation of Nod1-mediated intracellular signaling. Therefore, clinical application of this experimental model to develop new agents may succeed in attenuating inflammatory damage, and in this infectious process, may even decrease bacterial invasion of epithelial cells.

Our experiments identified the intracellular protein Nod 1 as being a major PRM involved in epithelial cell sensing of cagPAI(+) H. pylori bacteria. This statement is based on several lines of experimental evidence. First, we demonstrated that the Nod1-expressing AGS and Caco-2 cells responded specifically to stimulation with cagPAI(+) H. pylori strains. Secondly, we established the role of cagPAI as a virulence factor in gastric epithelial inflammation using cag-lacking model. Thirdly, we 
made certain that stable transfection of AGS and Caco-2 cells with a DN-Nod 1 construct inhibited $H$. pylori-induced NF$\kappa \mathrm{B}$ reporter activity in the cells. This indicates that DN Nod1transfected AGS cells be affected in their ability to mount proinflammatory responses to the bacterium. Finally, we showed that Nod1-mediated intracellular signaling pathway can elicit the neutrophil recruitment and migration across the epithelial layer. The gastric and colonic cell lines used in these studies are stably transfected and, in this regard, differ from normal gastric and colonic epithelial cells. However, we noted that despite their different origins, an identical array of proinflammatory cytokine was consistently upregulated in each of the cell lines in response to $H$. pylori infection.

In summary, we concluded that Nod 1 constitutively expressed by human gastric epithelial cells is essential for the activation of $\mathrm{NF}-\kappa \mathrm{B}$ and the upregulated production of important epithelial cell chemoattractants in a physiologic system in which human gastric epithelial cells are infected with $H$. pylori that bypasses TLR signaling. If the mechanism by which $H$. pylori activates the host innate immune system are addressed in detail, it should be possible to design novel therapies aimed at inhibiting or reducing the inflammation induced by this mucosal pathogen.

\section{CONFLICTS OF INTEREST}

No potential conflict of interest relevant to this article was reported.

\section{ACKNOWLEDGEMENTS}

The work was supported by the Korea Research Foundation Grant funded by the Korean Government (MOEHRD, Basic Research Promotion Fund) (KRF-314-2008-1-E00093).

We thank Ki Sung Kim for his excellent technical help.

\section{REFERENCES}

1. Smith MF Jr, Mitchell A, Li G, et al. Toll-like receptor (TLR) 2 and TLR5, but not TLR4, are required for Helicobacter pylori-induced NF-kappa B activation and chemokine expression by epithelial cells. J Biol Chem 2003;278:32552-32560.

2. Bäckhed F, Rokbi B, Torstensson E, et al. Gastric mucosal recognition of Helicobacter pylori is independent of Toll-like receptor 4. J Infect Dis 2003;187:829-836.

3. Rosenstiel P, Hellmig S, Hampe J, et al. Influence of polymorphisms in the NOD1/CARD4 and NOD2/CARD15 genes on the clinical outcome of Helicobacter pylori infection. Cell Microbiol 2006;8:1188-1198.

4. Olofsson A, Vallstrom A, Petzold K, et al. Biochemical and functional characterization of Helicobacter pylori vesicles. Mol Microbiol 2010;77:1539-1555.

5. Viala J, Chaput C, Boneca IG, et al. Nod1 responds to peptidogly- can delivered by the Helicobacter pylori cag pathogenicity island. Nat Immunol 2004;5:1166-1174.

6. Hofner P, Gyulai Z, Kiss ZF, et al. Genetic polymorphisms of NOD1 and IL-8, but not polymorphisms of TLR4 genes, are associated with Helicobacter pylori-induced duodenal ulcer and gastritis. Helicobacter 2007;12:124-131.

7. Kara B, Akkiz H, Doran F, et al. The significance of E266K polymorphism in the NOD1 gene on Helicobacter pylori infection: an effective force on pathogenesis? Clin Exp Med 2010;10:107-112.

8. Blaser MJ, Perez-Perez GI, Kleanthous H, et al. Infection with Helicobacter pylori strains possessing cagA is associated with an increased risk of developing adenocarcinoma of the stomach. Cancer Res 1995;55:2111-2115.

9. Censini S, Lange C, Xiang Z, et al. cag, a pathogenicity island of Helicobacter pylori, encodes type I-specific and disease-associated virulence factors. Proc Natl Acad Sci U S A 1996;93:14648-14653.

10. Keates S, Keates AC, Warny M, Peek RM Jr, Murray PG, Kelly CP. Differential activation of mitogen-activated protein kinases in AGS gastric epithelial cells by cag+ and cag- Helicobacter pylori. J Immunol 1999;163:5552-5559.

11. Covacci A, Rappuoli R. Helicobacter pylori: molecular evolution of a bacterial quasi-species. Curr Opin Microbiol 1998;1:96-102.

12. Kaparakis M, Turnbull L, Carneiro L, et al. Bacterial membrane vesicles deliver peptidoglycan to NOD1 in epithelial cells. Cell Microbiol 2010;12:372-385.

13. Magalhaes JG, Philpott DJ, Nahori MA, et al. Murine Nod1 but not its human orthologue mediates innate immune detection of tracheal cytotoxin. EMBO Rep 2005;6:1201-1207.

14. Chamaillard M, Hashimoto M, Horie $Y$, et al. An essential role for NOD1 in host recognition of bacterial peptidoglycan containing diaminopimelic acid. Nat Immunol 2003;4:702-707.

15. Jung HC, Eckmann L, Yang SK, et al. A distinct array of proinflammatory cytokines is expressed in human colon epithelial cells in response to bacterial invasion. J Clin Invest 1995;95:55-65.

16. Berin MC, Dwinell MB, Eckmann L, Kagnoff MF. Production of MDC/CCL22 by human intestinal epithelial cells. Am J Physiol Gastrointest Liver Physiol 2001;280:G1217-G1226.

17. Hase K, Eckmann L, Leopard JD, Varki N, Kagnoff MF. Cell differentiation is a key determinant of cathelicidin LL-37/human cationic antimicrobial protein 18 expression by human colon epithelium. Infect Immun 2002;70:953-963.

18. Kim JG, Lee SJ, Kagnoff MF. Nod1 is an essential signal transducer in intestinal epithelial cells infected with bacteria that avoid recognition by toll-like receptors. Infect Immun 2004;72:14871495.

19. Backert S, Schwarz T, Miehlke S, et al. Functional analysis of the cag pathogenicity island in Helicobacter pylori isolates from patients with gastritis, peptic ulcer, and gastric cancer. Infect Immun 2004;72:1043-1056.

20. Bauer B, Moese S, Bartfeld S, Meyer TF, Selbach M. Analysis of cell type-specific responses mediated by the type IV secretion system of Helicobacter pylori. Infect Immun 2005;73:4643-4652. 
21. Grubman A, Kaparakis M, Viala J, et al. The innate immune molecule, NOD1, regulates direct killing of Helicobacter pylori by antimicrobial peptides. Cell Microbiol 2010;12:626-639.

22. Gorrell RJ, Guan J, Xin Y, et al. A novel NOD1- and CagAindependent pathway of interleukin-8 induction mediated by the Helicobacter pylori type IV secretion system. Cell Microbiol 2013;15:554-570.

23. Allison CC, Ferrand J, McLeod L, et al. Nucleotide oligomerization domain 1 enhances IFN-gamma signaling in gastric epithelial cells during Helicobacter pylori infection and exacerbates disease severity. J Immunol 2013;190:3706-3715.
24. Oikawa T, Asano N, Imatani A, et al. Gene polymorphisms of NOD1 and interleukin-8 influence the susceptibility to erosive esophagitis in Helicobacter pylori infected Japanese population. Hum Immunol 2012;73:1184-1189.

25. Hidalgo IJ, Raub TJ, Borchardt RT. Characterization of the human colon carcinoma cell line (Caco-2) as a model system for intestinal epithelial permeability. Gastroenterology 1989;96:736-749.

26. Nash S, Stafford J, Madara JL. Effects of polymorphonuclear leukocyte transmigration on the barrier function of cultured intestinal epithelial monolayers. J Clin Invest 1987;80:1104-1113. 